\title{
Análisis de datos para el marketing digital emprendedor: Caso de estudio del Parque de Innovación Empresarial de Manizales
}

\author{
Juan Camilo Marín López* \\ Marcelo López Trujillo*
}

Fecha de recibido: 22 de agosto de 2018 Fecha de aprobado: 17 de agosto de 2019

Para citar: Marín López, J. C., \& López Trujillo, M. (2020). Análisis de datos para el marketing digital emprendedor. Caso de estudio del Parque de Innovación Empresarial de Manizales. Universidad \& Empresa, 22(38), 65-78. https://doi.org/10.12804/revistas.

urosario.edu.co/empresa/a.7135

* Administrador de Sistemas Informáticos, Facultad de Administración, Universidad Nacional de Colombia, Sede Manizales. Correo electrónico: jucmarinlo@unal.edu.co.

** Ingeniero de Sistemas. Magíster en educación. Doctor en Ingeniería Informática, Sociedad de la información y del conocimiento. Profesor del Departamento de Sistemas e Informática de la Universidad de Caldas, Catedrático del Departamento de Informática y Computación de la Universidad Nacional de Colombia, Sede Manizales. Correo electrónico: malopeztr@unal.edu.co. 


\title{
Resumen
}

El marketing digital o e-marketing ofrece diferentes oportunidades y beneficios que pueden ser aprovechadas por las pymes para mejorar su eficiencia y competitividad. Sin embargo, el desafío real es analizar los datos recopilados y utilizarlos para optimizar procesos y recursos. A pesar de la considerable importancia que se le ha dado a este tema, se han realizado pocas investigaciones que unan los conceptos de e-marketing y análisis de datos en el contexto emprendedor. Este estudio se propuso explorar y describir el panorama del análisis de datos para el marketing digital en el Parque de Innovación Empresarial de Manizales, mediante la realización de 20 encuestas. En esta exploración, se encontró que los microempresarios reconocen la importancia y utilizan el marketing digital, pero solo la mitad de ellos realiza un análisis de datos superficial o básico, debido a diversas limitaciones que se expondrán. Se abren nuevas puertas para futuros estudios que tengan un enfoque de choque frente a la realidad encontrada.

Palabras clave: análisis de datos, marketing digital, pymes.

\section{Data Analysis for Entrepreneurial Digital Marketing: Case Study Business Innovation Park - Universidad Nacional, Manizales}

\begin{abstract}
Digital marketing offers different opportunities and benefits that SMEs can take advantage of to improve their efficiency and competitiveness. Nonetheless, the real challenge is to analyze the compiled data and utilize it to optimize processes and resources. Despite its importance, there are little researches linking e-marketing and entrepreneurial to data analysis. This paper will explore and describe the data analysis landscape for digital marketing in the Business Innovation Park - Universidad Nacional, Manizales, through 20 surveys. The exploration and description showed that micro-entrepreneurs acknowledges the importance and make use of digital marketing. However, only half of them analyze external or essential data due to various limitations. New doors open for future studies that have a shock approach to the reality found.
\end{abstract}

Keywords: Data analysis, digital marketing, sMEs.

\section{Análise de dados para o marketing digital empreendedor: Caso de estudo Parque de Inovação Empresarial - Universidad Nacional Sede Manizales}

\section{Resumo}

O marketing digital oferece diferentes oportunidades e benefícios que podem ser aproveitadas pelas pME para melhorar sua eficiência e competitividade. No entanto, o desafio real é analisar os dados recolhidos e utilizá-los para otimizar processos e recursos. Apesar da considerável importância que se lhe tem reconhecido a isto, se tem realizado poucas pesquisas que unam os conceitos de e-marketing e análise de dados no contexto empreendedor. Este estudo se propõe explorar e descrever o panorama da análise de dados para o marketing digital no Parque de Inovação Empresarial de Manizales, mediante a realização de 20 enquetes. Se encontrou que os microempresários reconhecem a importância e utilizam o marketing digital, mas só a metade deles realiza uma análise de dados superficial ou básica devido a diversas limitações que expomos. Se abrem novas portas para futuros estudos que tenham um enfoque de choque frente à realidade encontrada.

Palavras-chave: análise de dados, Marketing digital, PME. 


\section{Introducción}

Con la llegada y la evolución del internet, las personas y las empresas han cambiado la forma de convivir y comunicarse. Esto se ha evidenciado en las versiones que han ido transformando la web. En los años noventa se creó la web 1.0; en 2004 ya se hablaba de la web 2.0, la cual permite al usuario interactuar en el desarrollo y expansión de las páginas, generando así la web social; solamente 2 años después, ya se mencionaba la web 3.0 y, actualmente, ya se piensa en la versión 4.0, donde la web se conecte aún más con la vida real (Martínez-López, Anaya \& Aguilar, 2015), dando paso a que se hable de web x.0 para incluirlas a todas y a las futuras.

El marketing no ha sido ajeno al continuo cambio digital, y ha aprovechado las ventajas que ofrecen las herramientas tecnológicas para acercarse a sus clientes, con lo que se generó el marketing digital. Es por esto que también ha evolucionado en paralelo a su versión 2.0, conocida también como Social Media Marketing (sмm), la cual se interesa en el cliente y la interacción con él al utilizar los canales del sмм en lugar de la televisión, prensa o radio (Fuentes, Huacón \& Arisendy, 2015).

Gracias a la web 2.0 y al marketing 2.0, la comunicación empresa-cliente ahora es bidireccional, lo que permite una retroalimentación más activa y directa, y agiliza la toma de decisiones por parte de las organizaciones. Las empresas pequeñas pueden sacar provecho de esto y además usar las herramientas de las redes sociales para mejorar su reputación, fortalecer su marca y adelantarse o responder a los comentarios de clientes, proveedores $\mathrm{u}$ otras partes interesadas. Esto ha cambiado la forma en la que los empresarios obtienen

y distribuyen información, e igualmente ha enriquecido la experiencia del servicio de la pequeña empresa y contribuido al proceso de creación de valor (Jones, 2010; Kannan \& Li, 2017).

Además, el uso de las cuentas de usuario y su permanente interacción permite a las plataformas publicitarias crear un perfil detallado de los intereses, gustos, hábitos y otras características de los usuarios (Guha, Cheng \& Francis, 2011). De este modo, se facilita realizar una detallada segmentación, debido a la facilidad de rastrear un usuario o grupo en particular, de acuerdo con sus intereses y comportamientos. Esto lógicamente ha 
abierto una discusión sobre la privacidad de las personas al usar los diferentes elementos tecnológicos ( Newman, Chang, Walters \& Wills, 2016).

En este mundo digital, los grandes datos se han convertido en la norma y en la forma de acertar en la toma de decisiones. Es por esto que el desafío más importante del e-marketing es la capacidad de generar y aprovechar la información profunda recopilada de los clientes, ya que un seguimiento y análisis eficiente del comportamiento del usuario es un requisito clave para optimizar las campañas publicitarias y los presupuestos (Leeflang, Verhoef, Dahlström \& Freundt, 2014). El big data y la minería de datos cumple un papel importante en estos casos, al ayudar a las organizaciones a descubrir el conocimiento oculto en la enorme cantidad de datos recolectados (Turban, Sharda \& Delen, 2011).

A pesar de que existen estudios de la importancia del e-marketing para las pymes, y de la importancia del análisis de datos para las organizaciones, no existen estudios que unan estos dos conceptos, aplicados en el contexto de empresas de pequeño y mediano tamaño. Y menor ha sido el estudio de esto en Colombia. Por ello, con este artículo se pretende examinar la unión de todas estas temáticas y evaluar cómo se comportan en el caso de estudio establecido.

El artículo se organiza de la siguiente manera: la sección siguiente describe la problemática en particular a resolver y destaca la importancia de la investigación; luego, se describe brevemente la metodología utilizada. Finalmente, se exponen los resultados presentados y las conclusiones a las que estos conllevan, lo que da pie para la formulación de futuras investigaciones.

\section{Problemática}

\subsection{Definición del problema y justificación}

De acuerdo con varios estudios, en el año 2018 hay más de 4 mil millones de personas en todo el mundo que usan internet. En Colombia, los usuarios de internet alcanzan el $63 \%$ de la población total, lo que equivale a cerca de 31 millones, número que también 
representa la cantidad de usuarios activos en las redes sociales. De esta cantidad de usuarios, el $88 \%$ se conecta todos los días desde cualquier dispositivo. Sin embargo, de las personas de la población mayores a 15 años, solamente el 6\% realizan compras y/o pagos online (We Are Social, 2018).

Esto quiere decir que, en el mundo, y a nivel nacional, existe un número significativo de usuarios online; sin embargo, los mecanismos del marketing digital, como el e-commerce, aún tienen muchísimo campo para crecer. Este terreno prometedor puede ser aprovechado por las pymes, ya que les permite lograr un alcance mucho mayor mediante estas plataformas, sin la necesidad de realizar una enorme inversión. Sin embargo, las pymes no utilizan todo el potencial de estas nuevas herramientas digitales (Taiminen \& Karjaluoto, 2015). En Colombia, solamente el 1\% de las pymes tiene adopción total del marketing digital. Esto fue hallado mediante una investigación liderada por Sandra Rojas (2017), profesora de la Facultad de Ciencias Económicas de la Universidad Nacional de Colombia, quien además afirma:

El desarrollo tecnológico y las nuevas redes de comunicación cambiaron sustancialmente el comportamiento de los consumidores y la forma como las pymes deben enfrentar el entorno comercial. Sin embargo, en Colombia es incipiente la adopción de las nuevas tecnologías de información para crear valor y estructurar campañas de marketing digital (p. 1).

Esta cifra del $1 \%$ es alarmante, más si se tiene en cuenta que el desafío no es solo la implementación de estrategias de e-marketing, sino poder analizar los datos recopilados del cliente para mejorar y optimizar procesos (Leeflang et al., 2014). Esto proporciona la oportunidad de realizar CRM analítico (Customer Relationship Management o Gestión de Relaciones con los Clientes), el cual podría ayudar a una empresa a discriminar mejor y asignar recursos de una forma más eficaz al grupo de los consumidores más rentables, por medio del uso de técnicas de análisis de datos (Ngai, Xiu \& Chau, 2009). Realizar este tipo de procesos es un factor diferencial clave para las pymes, y más si se tiene en cuenta que las empresas que analizan sistemáticamente los datos superan a sus competidores (Davenport, 2006). Sin embargo, la incapacidad de descubrir información valiosa y oculta en los datos impide que las organizaciones transforman estos datos en conocimiento valioso y útil (Berson, Smith \& Thearling, 2000). 
El análisis de datos se ha convertido en algo cada vez más importante en los últimos años, no obstante, no se conoce hasta qué punto las empresas ya están aplicando análisis o ciencia de datos. Esto, debido a que hoy en día hay una gran cantidad de desafíos, los cuales incluyen la captura, limpieza, almacenamiento, búsqueda, intercambio, transferencia, análisis y visualización de los datos (Snijders, Matzat \& Reips, 2012). Además, se ha encontrado que el análisis tiene muchas oportunidades inexploradas en el campo de las redes sociales (Lismont, Vanthienen, Baesens \& Lemahieu, 2017).

Es el caso de los árboles de decisión y la regresión logística, dos algoritmos muy populares en la predicción de abandono del cliente con un fuerte rendimiento predictivo y una buena comprensión del fenómeno (De Caigny, Coussement \& De Bock, 2018). Para el análisis predictivo y prescriptivo se utiliza también programación lineal como un enfoque para la toma de decisiones en ventas, (Bischhoffshausen, Paatsch, Reuter, Satzger \& Fromm, 2015).

Por todo lo anterior, es de gran relevancia conocer el estado de la aplicación del análisis de datos a la información recopilada desde el marketing digital en todas las empresas, especialmente en las de pequeño y mediano tamaño. Ya que, en estas, cualquier elemento diferenciador es clave para obtener ventajas competitivas en el mercado, y el e-marketing ofrece muchas posibilidades para esto. Del mismo modo, se debe considerar también la protección de los datos y la forma en que los usuarios autorizan el uso de su información.

En Colombia, la competitividad de las pymes es un tema relevante, ya que aportan el $37 \%$ del Producto Interno Bruto (PIB) Nacional. Por ejemplo, entre 2009 y 2017, cerca del 96\% de los registros en la Cámara de Comercio de Bogotá fueron de este tipo de empresas (Rojas, 2017). Sin embargo, si se habla de emprendimiento en Colombia, se debe nombrar a Manizales, ya que es considerada rey [sic] del emprendimiento (Semana, 2015). Esto lo ha logrado gracias a diferentes programas promovidos desde el gobierno. Como "Manizales más” y "La ruta del emprendimiento" (Finanzas Personales, 2013).

La Universidad Nacional de Colombia, Sede Manizales, ha cumplido un papel importante para que Manizales sea catalogada de esta forma, debido a que ha participado activamente en la mayoría de los programas de emprendimiento de la zona. Además, mediante el Consultorio Administrativo y el Parque de innovación Empresarial (PIE), se ha vinculado 
año tras año con proyectos que surgen en la región para fomentar el emprendimiento (Universia Colombia, 2013).

Ofrecer espacios donde se fomenten la innovación para la competitividad, la investigación aplicada, la transferencia de tecnología y la formación continuada son los principales objetivos del Parque de Innovación Empresarial. Este lleva alrededor de 13 años de funcionamiento, constituyéndose en un espacio importante para el acompañamiento y fomento de las iniciativas empresariales en el sector (Revista Mprende, 2016).

Por lo tanto, si se quiere conocer y evaluar el contexto actual del análisis de datos que se realiza a la información proveniente del e-marketing en las pymes de la zona, el PIE es un lugar indicado para ello. Esto, debido a que en este lugar se encuentran aproximadamente 50 empresas de pequeño tamaño, en las cuales sería de gran interés conocer cómo es dicho panorama para estos empresarios, ya que actualmente no existen estudios del tema de interés en aquel lugar, ni en la región. Teniendo en cuenta, además, que es difícil encontrar estudios en donde se vean unidos los conceptos de análisis de datos para el marketing digital, enmarcado en el panorama de las pymes.

La realización de la investigación permite conocer dos aspectos relevantes para las pymes, y cómo se relacionan entre estos. El primero, es el grado de uso del e-marketing en este segmento de empresas, lo que permite conocer qué tan relevante es el uso de este y qué herramientas o elementos principales se están empleando. En segundo lugar, se identificaron las técnicas y programas a los que están acudiendo las empresas para realizar análisis de datos de la información recolectada. Igualmente, se conoció la relación que existe hoy entre estos dos factores, concluyendo en el estado actual del análisis de datos en el marketing digital emprendedor, acotado en el PIE. Ya conociendo el panorama real, se permite evaluar dicho contexto, con el fin de emitir el grado de madurez del análisis de datos de acuerdo con modelos teóricamente propuestos.

\subsection{Antecedentes}

El marketing ha sido un interés y una preocupación central de la investigación emprendedora en todo el mundo por muchos años (Carson, Cromie, McGowan \& Hill, 1995). Es por esto que han surgido ramas dedicadas a este segmento en específico, como el marketing emprendedor (Marín-López, Robledo \& Duque-Méndez, 2017). Debido a la 
importancia que esto ha venido teniendo, Eid y El-Gohary (2013) evaluaron la influencia del $e$-marketing en las pymes, encontrando que el uso de este tipo de herramientas tiene una influencia positiva en las pequeñas y medianas empresas.

Para la adopción del marketing digital es necesario implementar diferentes tecnologías, elemento que es vital para la supervivencia de las pequeñas empresas. Es por esto que Alford y Page (2015) realizaron una investigación en la cual descubrieron que había un fuerte interés de este tipo de empresas por la adopción de la tecnología para el marketing $\mathrm{y}$, adicionalmente, reconocían sus beneficios relacionados. Sin embargo, la capacidad de aprovechar estas oportunidades se vio limitada por la falta de conocimiento y por la incapacidad de medir el rendimiento de la inversión.

En el mismo año, Dervan (2015) evaluó el uso y la aplicación del big data por empresas que participan activamente en las redes sociales y contienen grandes conjuntos de datos. En este estudio, se aprobó que las empresas obtienen un mayor ROI en redes sociales por el uso del big data, al utilizar herramientas analíticas avanzadas para el procesamiento de los datos. Con esto logran describir y predecir las características de los usuarios, utilizando esto para orientar, programar, adaptar y activar la publicación de contenidos o anuncios convincentes para los usuarios. Además, abrió puertas para futuras investigaciones que exploren el uso de los datos de las redes sociales, promoviendo el presente estudio.

Deep Learning y Big Data Analytics son dos puntos focales de la ciencia de datos, los modelos de aprendizaje profundo han permitido avances importantes en el reconocimiento de voz y la visión por computadora, en el reconocimiento de patrones y toma de decisiones por predicciones, para las organizaciones que necesitan recopilar una gran cantidad de datos, tomados de la red social y del entorno del IoT, uno de los mayores activos es usar el Deep Learning para analizar una gran cantidad de datos (Sohangir, Wang, Pomeranets \& Khoshgoftaar, 2018).

Sin embargo, los investigadores han encontrado que la aplicación real de análisis en las empresas todavía está en sus etapas iniciales. Es por esto que Lismont et al. (2017) estudiaron la aplicación del análisis de datos en múltiples compañías. Allí, se encontró que las empresas que comenzaron tempranamente con la aplicación de análisis, ahora 
aplican técnicas más complejas como redes neuronales, y aplicaciones más avanzadas, como análisis de recursos humanos y análisis predictivo.

Existen diversas posibilidades de aplicación del análisis de datos utilizando computación cognitiva, el caso del Deep Reinforcement Learning, representado como un proceso de decisión de Markov (MDP), que parte del supuesto de que el agente de marketing está situado en un entorno y está representado por un cierto estado, el agente puede realizar ciertas acciones en el entorno, luego recibe la recompensa y transforma el entorno en un nuevo estado, (Bischhoffshausen et al., 2015; Zhang, Yao \& Guan, 2017).

Los sistemas de recomendación y análisis de sentimientos y caracterización de leads son vitales para la comercialización y el marketing aplicando algoritmos de aprendizaje extremo coevolutivo, lógica difusa y prescripción por análisis de valores, (Lau, Zhang \& $\mathrm{Xu}, 2017)$.

El estudio que se presenta en este artículo permitió indicar la etapa de madurez analítica de las empresas, descubriendo cuatro conglomerados: 1) sin análisis, 2) desarrolladores de análisis, 3) adoptantes de analítica sostenible e 4) innovadores analíticos disruptivos. Esta categorización es la que se empleó en el desarrollo del presente trabajo, con el fin de dar un concepto del grado de madurez analítica de las empresas del PIE.

\section{Metodología}

El propósito del estudio realizado, el cual es de tipo exploratorio y descriptivo, se basa en analizar la aplicación del análisis de datos para el marketing digital, en el Parque de Innovación Empresarial de Manizales. Esto se logró mediante una investigación de campo en dicha entidad, por medio de la realización de 20 encuestas que permitieron reconocer y describir las técnicas, herramientas y retos en el objeto de estudio.

Para lo anterior, primero se elaboró el cuestionario de acuerdo con las variables necesarias identificadas para los fines del proyecto. Posteriormente, se solicitó la base de datos de las empresas pertenecientes al parque con la persona a cargo de este. De allí, 
se recibieron los datos generales de 52 empresas, de las cuales 47 contaban con correo electrónico. A estas 47 empresas se les envió el cuestionario por medio electrónico, de los cuales se recibieron 20 respuestas. Finalmente, se procedió a analizar la información recopilada por el instrumento empleado, hallando las conclusiones de la siguiente sección.

\section{Resultados y análisis}

Las empresas que aportaron al estudio pertenecen a múltiples sectores empresariales, y no sobrepasan los 12 empleados, se debe considerar que el $60 \%$ solo lleva entre 1 y 5 años en el mercado. De estas empresas, el $55 \%$ vende sus productos tanto online, como offline, y el 35\% únicamente por medio físico. Se halló que más de la mitad (55\%) de las empresas consideran muy importante, casi vital, el uso del marketing digital y solo $5 \%$ la consideran poco importante. Debido a esta perspectiva positiva del marketing digital, el $70 \%$ ha invertido en sus elementos y el $10 \%$ está en planes de hacerlo. Se identificaron los 5 elementos principales que utilizan para tener presencia web, los cuales son en su orden: sмм orgánico (85\%), página web informativa (70\%), email marketing (65\%), content marketing (55\%) у sмм pago (55\%); destacándose que ninguna de las empresas utiliza aplicaciones móviles para llegar a sus usuarios. Igualmente, se encontró que los empresarios ya están familiarizados con los diferentes elementos de presencia digital, ya que el 75\% reconoció todos o la mayoría por los que se consultó.

En cuanto al análisis de datos del e-marketing realizado, se encontró que un $50 \%$ los analiza de alguna forma, un $30 \%$ lo consulta en plataformas y un $25 \%$ lo almacena. Los análisis que realizan son principalmente de tipo estadístico (47.4\%), mientras que los menos utilizados son el análisis semántico (15.8\%) y el machine learning (15.8\%). El objetivo principal con el que realizan este tipo de procesos es para fines de mercadeo (70\%) y el menor es para recursos humanos (15\%). Se identificó que las técnicas más empleadas son la integración de datos de diferentes fuentes (26.3\%) y clustering o segmentación (21.1\%). Actualmente, no se aplican técnicas avanzadas como redes neuronales, cubos OLAP, máquinas vectoriales, entre otros. Las herramientas más utilizadas son las herramientas ofimáticas como las hojas de cálculo (68.4\%), plataformas publicitarias (57.9\%) y herramientas de análisis web (42.19\%). 
En cuanto a los beneficios, todos los que han realizado análisis de datos al marketing digital han percibido ventajas de algún tipo, iniciando con la percepción de un mayor reconocimiento de marca (45\%), incremento de conocimiento para la organización (35\%), mejor reputación (35\%) y ventajas competitivas (35\%). Mientras que los obstáculos para la implementación, se atribuyen principalmente a limitaciones en recursos o habilidades (65\%).

Las empresas que venden sus productos física y virtualmente tienen un mayor conocimiento, conciencia e inversión en el e-marketing, pero pocos realizan análisis con los datos. Los que consideran muy importante la implementación del e-marketing y realizan inversión en este, tienden a analizar más los datos o realizar acciones con estos. Se hallaron mejores resultados en las empresas relacionadas con el sector TIC y el sector publicitario.

\section{Conclusiones}

En general, el panorama encontrado muestra que las pymes han empezado a reconocer la importancia y los beneficios del marketing digital y por esto lo han empezado a aplicar. Sin embargo, solo la mitad realiza análisis de datos de esto, aclarando que el análisis aplicado es del más básico, utilizando por ejemplo hojas de cálculo. Estas empresas ven en la limitación de recursos y habilidades los principales obstáculos para realizar los análisis. No es posible generalizar el grado de madurez del análisis de datos (Lismont et al., 2017), ya que algunas empresas se encuentran en la categoría de "sin análisis" y otras en "desarrolladores de análisis" en su forma más básica.

A la falta de formación y entrenamiento en análisis de datos (en técnicas para descripción, diagnóstico, predicción y prescripción) se suman los aspectos de seguridad de la información y protección de datos, de manera que la calidad de los datos internos y externos y su uso sean garantizados tanto para las empresas como para los potenciales clientes, como lo propone, ( Li, Li, Ye, Li, Chen \& Xiang, 2018). La evolución de la computación cognitiva está permitiendo y permitirá cada vez con mayor incidencia una mejor toma de decisiones, gracias a los avances de las ciencias de los datos y su aplicación a la comercialización y el marketing. 
Este estudio abre nuevas puertas para futuras investigaciones que tengan un enfoque explicativo de la realidad encontrada. Se podrían realizar investigaciones que reconozcan los factores influyentes en el campo estudiado y se podrían medir y comparar diferentes métodos o procedimientos que estén afectando los resultados. Igualmente, sería relevante idear trabajos para la concientización, capacitación y/o enseñanza de este tipo de conceptos, para las empresas que obtuvieron resultados negativos. La investigación permite, además, que la universidad y los integrantes del PIE tomen acciones de acuerdo con los hallazgos realizados, que permitan fortalecer sus habilidades en el tema y lograr una mayor competitividad. Por otro lado, ayudó a promover y a destacar la importancia de la unión de los conceptos estudiados, como también resaltar la importancia que tienen y la aplicación de estos en el contexto de las pymes.

\section{Referencias}

Alford, P. \& Page, S. J. (2015). Marketing technology for adoption by small business. The Service Industries Journal, 35(11-12), 655-669.

Berson, A., Smith, S., \& Thearling K. (2000). Building Data Mining Applications for CRM. New York: McGraw-Hill.

Bischhoffshausen, J. K. von, Paatsch, M., Reuter, M., Satzger, G., \& Fromm H. (2015). An Information System for Sales Team Assignments Utilizing Predictive and Prescriptive Analytics. In 2015 IEEE 17th Conference on Business Informatics. IEEE, Lisboa. https://doi. org/10.1109/cbi.2015.38

Carson, D., Cromie, S., McGowan, P., \& Hill, J. (1995). Marketing and Entrepreneurship in SMEs: An Innovative Approach. Harlow: Pearson Education.

Davenport, T. H. (2006). Competing on Analytics. Harvard Business Review 84(1), 98-107.

De Caigny, A., Coussement, K., \& De Bock. K. W. (2018). A New Hybrid Classification Algorithm for Customer Churn Prediction Based on Logistic Regression and Decision Trees. European Journal of Operational Research, 269(2), 760-772.

Eid, R. \& El-Gohary, H. (2013). The impact of E-marketing use on small business enterprises' marketing success. The Service Industries Journal, 33(1), 31-50.

Finanzas Personales (2013). Manizales le apuesta a ser la ciudad del emprendimiento. Finanzas Personales. Recuperado de http://www.finanzaspersonales.co/ultimas-noticias/articulo/ manizales-apuesta-ciudad-del-emprendimiento/51292 
Fuentes V. S. L., Huacón, V., \& Arisendy, A. (2015). Análisis de la evolución del marketing 1.0, al marketing 3.0 y propuesta de aplicación en la marca miller de la compañía Cervecería Nacional en el sector de la ciudad de Guayaquil. (Tesis de grado, Universidad Politécnica Salesiana, Guayaquil, Ecuador). Recuperado de https://dspace.ups.edu.ec/ handle/123456789/10026.

Guha, S., Cheng, B., \& Francis, P. (2011). Privad: Practical Privacy in Online Advertising. In USENIX Conference on Networked Systems Design and Implementation, ACM SIGCOMM and ACM SIGOPS, Boston.

Jones, B. (2010). Entrepreneurial Marketing and the Web 2.0 Interface. Journal of Research in Marketing and Entrepreneurship, 12(2), 143-152.

Kannan, P. K. \& Li, A. (2017). Digital Marketing: A Framework, Review and Research Agenda. International Journal of Research in Marketing, 34(1), 22-45.

Lau, R. Y. K., Zhang, W., \& Xu, W. 2017. Parallel Aspect-Oriented Sentiment Analysis for Sales Forecasting with Big Data. International Journal of Operations \& Production Management. https://doi.org/10.1111/poms.12737

Leeflang, P. S. H., Verhoef, P. C., Dahlström, P., \& Freundt, T. (2014). Challenges and Solutions for Marketing in a Digital Era. European Management Journal, 32(1), 1-12.

Li, P., Li, T., Ye, H., Li, J., Chen, X., \& Xiang, Y. (2018). Privacy-Preserving Machine Learning with Multiple Data Providers. Future Generations Computer Systems: FGCS, 87, 341-350.

Lismont, J., Vanthienen, J., Baesens, B., \& Lemahieu, W. (2017). Defining Analytics Maturity Indicators: A Survey Approach. International Journal of Information Management, 37(3), 114-124.

Marín-López, J. C., Robledo, J. S., \& Duque-Méndez, N. (2017). Marketing Emprendedor: Una Perspectiva Cronológica Utilizando Tree of Science. Revista Civilizar de Empresa Y Economía, 13(1), 113-123.

Martínez-López, F. J., Anaya, R., Aguilar, R., \& Molinillo, S. (2015). Online Brand Communities: Using the Social Web for Branding and Marketing. Berlín: Springer.

Newman, R., Chang, V., Walters, R. J., \& Wills, G. B. (2016). Web 2.0-The Past and the Future. International Journal of Information Management, 36(4), 591-598.

Ngai, E. W. T., Xiu, L., \& Chau, D. C. K. (2009). Application of Data Mining Techniques in Customer Relationship Management: A Literature Review and Classification. Expert Systems with Applications, 36(2, Part 2), 2592-2602.

Revista Mprende (2016). Manizales le apuesta a la innovación empresarial. Revista Mprende. Recuperado de http://mprende.co/emprendimiento/manizales-le-apuesta-la-innovaci\%C3\%B3n-empresarial 
Rojas, S. (2017). Solo $1 \%$ de Las pymes tiene adopción total del marketing digital. Bogotá: Universidad Nacional de Colombia. Recuperado de http://www.fce.unal.edu.co/sala-de-prensa/noticias/todas-las-noticias/949-solo-1-de-las-pymes-tiene-adopcion-total-delmarketing-digital.html

Semana (2015). Manizales, Rey Del Emprendimiento. Foros Semana Manizales, Rey Del Emprendimiento. Recuperado de https://www.semana.com/nacion/articulo/foros-semana-manizales-rey-del-emprendimiento/440454-3

Snijders, C., Matzat, U., \& Reips, U. D. (2012). 'Big Data': Big Gaps of Knowledge in the Field of Internet Science. International Journal of Internet Science, 7(1), 1-5.

Sohangir, S., Wang, D., Pomeranets, A., \& Khoshgoftaar, T. M. (2018). Big Data: Deep Learning for Financial Sentiment Analysis. Journal of Big Data, 5(1). https://doi.org/10.1186/ s40537-017-0111-6

Taiminen, H. M. \& Karjaluoto, H. (2015). The Usage of Digital Marketing Channels in SMEs. Journal of Small Business and Enterprise Development, 22(4), 633-651.

Turban, E., Sharda, R., \& Delen, D. (2011). Decision Support and Business Intelligence Systems. India: Pearson Education.

Universia Colombia (2013). La UN en Manizales se encuentra a la vanguardia en emprendimiento e innovación. Noticias Universia Colombia. Recuperado de http://noticias.universia.net.co/vida-universitaria/noticia/2013/11/15/1063895/manizales-encuentra-vanguardia-emprendimiento-e-innovacion.html

We Are Social. (2018). Digital in 2018 in Southern America Part 2 - South. SlideShare. Recuperado de https://es.slideshare.net/wearesocial/digital-in-2018-in-southern-america-part-2-south-86863967

Zhang, Y., Yao, J., \& Guan, H. (2017). Intelligent Cloud Resource Management with Deep Reinforcement Learning. IEEE Cloud Computing, 4(6), 60-69. 